\title{
Combining Fine Needle Aspiration With Brushing Cytology Has Improved Yields in Diagnosing Pancreatic Ductal Adenocarcinoma
}

\author{
Xin Jing, M.D., ${ }^{1 *}$ Erik-Jan Wamsteker, M.D., ${ }^{2}$ Hong Li, M.D., ${ }^{1}$
} and Robert T. Pu, M.D., Ph.D. ${ }^{1}$

The aim of this retrospective study is to evaluate the diagnostic yields of combining fine needle aspiration (FNA) with brushing cytology $(B C)$ in clinical work-up of pancreatic ductal adenocarcinoma.

The study included a total of 97 patients who underwent both FNA and BC along with histologic/clinical follow-up $(F / U)$. Cytologic diagnoses were categorized as negative for neoplasm (NEG), atypicallfavor neoplasm (AN), and suspicious or positive for neoplasm (POS). Based on the cytologic diagnoses, the cohort was divided as follows: 23 had concordant FNA and BC diagnoses of POS/AN, all were neoplasms on $F / U ; 34$ had disconcordant (POS/AN vs. NEG) FNA and BC diagnoses, all but 2 were neoplasms on $\mathrm{F} / \mathrm{U}$; The remaining 40 were $N E G$ on both $F N A$ and $B C, F / U$ revealed that 10 were neoplasms and 30 were chronic pancreatitis. Overall, FNA rendered more true positive diagnoses than BC. However, BC but not FNA detected neoplasms in 10 patients. Most of the neoplasms identified on F/ $U$ were ductal adenocarcinoma (59 of 65). Diagnostic sensitivity, specificity, positive predictive value, negative predictive value and accuracy were 69.2, 93.8, 95.7, 60, and 77.3\% for FNA alone, 50.8, 100, 100, 50.0, and 67.0\% for BC alone, and 84.6, 100, 100, 76.2, and $89.7 \%$ for combining FNA with BC. In conclusion, both EUS-guided FNA and BC are valuable modalities in the preoperative diagnosis of pancreatic ductal adenocarcinoma. When used in combination, the two modalities complement each other and achieve better diagnostic yield in pancreatic ductal adenocarcinoma than either FNA or BC alone. Diagn. Cytopathol. 2009;37:574-578. @ 2009 Wiley-Liss, Inc.

\footnotetext{
${ }^{1}$ Department of Pathology, University of Michigan Health System, Ann Arbor, Michigan

${ }^{2}$ Department of Internal Medicine, University of Michigan Health System, Ann Arbor, Michigan

*Correspondence to: Xin Jing, M.D., Department of Pathology, University of Michigan Health System, 1500 E. Medical Center Drive, Ann Arbor, MI 48108. E-mail: xinjing@med.umich.edu

Received 29 October 2008; Accepted 16 January 2009

DOI 10.1002/dc.21062

Published online 20 March 2009 in Wiley InterScience (www. interscience.wiley.com).
}

Key Words: EUS-guided fine needle aspiration; duct brushing; pancreatic lesions; pancreatic ductal adenocarcinoma

Both brushing cytology (BC) and EUS-guided fine needle aspiration (FNA) have been widely recognized as a wellestablished modality for diagnosing pancreatiobiliary neoplasms. Several large studies showed modest sensitivity of $\mathrm{BC}$, ranging from 44 to $55 \% .^{1-4}$ A considerable variation in diagnostic yield of EUS-guided FNA has been documented in several studies from different institutions ${ }^{5-10}$ and one multicenter retrospective study. ${ }^{11}$ In this regard, many factors played a role such as size, location and characteristics (i.e., well vs. ill-defined, solid vs. cystic) of neoplasms as well as other technical matters involved. Between 1996 and 2006, the number of EUS-guided FNA performed in our institution increased dramatically (42155/year) and diagnostic sensitivity and accuracy (unpublished) were compatible with that reported by other groups. To our knowledge, there was limited data regarding combination of both FNA and BC for diagnosing pancreatic neoplasms. The aim of this study was to evaluate the diagnostic efficacy of combining EUS-guided FNA with BC in clinical work-up of pancreatic neoplasms, particularly pancreatic ductal adenocarcinoma.

\section{Materials and Methods}

This was a retrospective study approved by the Institutional Review Board at the University of Michigan. Patients who underwent both EUS-guided FNA and BC during their clinical work-up for suspected pancreatic neoplasms in our institution were included in this study. The cases were retrieved through a search of the departmental database in the period of 1996-2006. The data including 


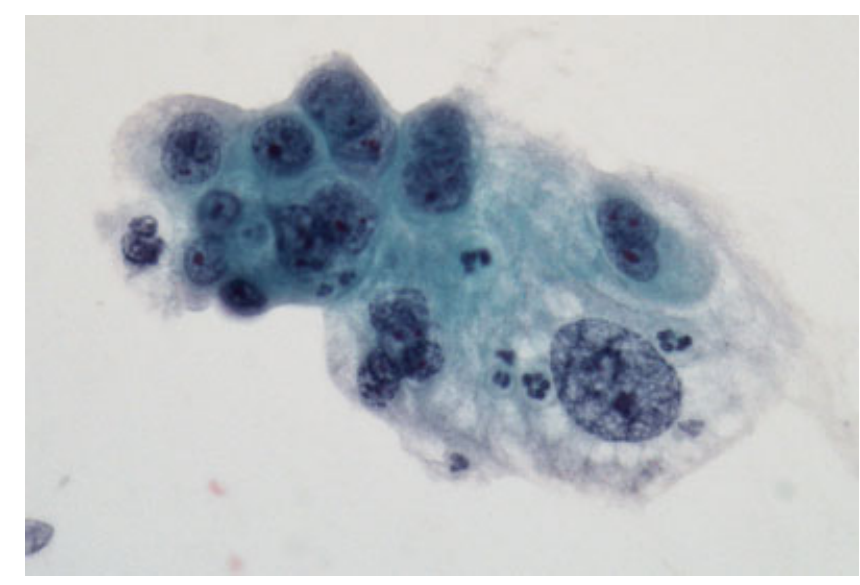

Fig. 1. Example of AN diagnosed on BC. This is the only cluster of atypical cells which show nuclear enlargement with high nuclear/cytoplasmic ratio, irregular nuclear membranes, coarse chromatin and prominent nucleoli (Papanicolaou stain, $\times 400$ ). [Color figure can be viewed in the online issue, which is available at www.interscience.wiley.com.]

age and gender of patients, size and location of lesions, numbers of EUS-guided FNA and BC performed on individual patients, methods of sample preparation and cytologic diagnoses were collected. Multiple samples obtained from one patient were treated as one case in this study. For EUS-guided FNA, eight gastrointestinists with similar experience performed the procedures and the total number of passes ranged between 4 and 10. Two direct smears were made from each pass and the needle was then rinsed in Cytolyt ${ }^{\circledR}$ solution (Cytyc Corporation, Marlborough, MA) for a ThinPrep ${ }^{\circledR}$ and/or cell block. One smear was air-dried, stained with Diff-Quik stain and then evaluated immediately for specimen adequacy. A preliminary diagnosis was rendered when possible. The other smear was quickly fixed with Sprayfix ${ }^{\mathrm{TM}}$ and stained with Papanicolaou stain. For BC, two direct smears were made, quickly fixed with Sprayfix $^{\mathrm{TM}}$ and stained with Papanicolaou stain. In some cases, the brushes were submitted in Cytolyt ${ }^{\mathrm{R}}$ solution (Cytyc Corporation, Marlborough, MA) and a ThinPrep ${ }^{\circledR}$ slide was then prepared.

Cytologic diagnoses were divided into the following three categories: negative for neoplasm (NEG) including benign and reactive atypia, atypical/favor neoplasm (AN), and suspicious or positive for neoplasm (POS). Representative examples of AN and POS are shown in Figures 1 and 2, respectively. For the purpose of data analysis, AN was considered a positive diagnosis.

Follow-up (F/U) information was available for all individuals; including surgical biopsy/excision diagnoses, as well as radiological imaging and/or clinical course/outcome when histopathological examination was not available. The maximum period of $\mathrm{F} / \mathrm{U}$ is 9 years. Using the cytologic and histologic diagnoses, as well as the $\mathrm{F} / \mathrm{U}$

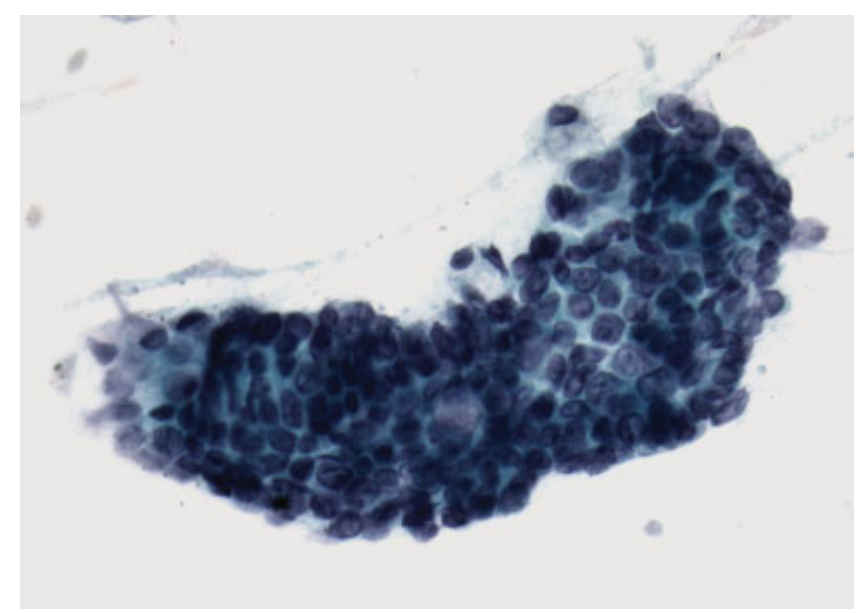

Fig. 2. Example of POS on EUS-guided FNA. A group of atypical cells showing a "drunken honeycomb" appearance with loss of cellular polarity and crowding. Nuclear enlargement with high nuclear/cytoplasmic ratio and vesicular nuclei with irregular nuclear membranes. Prominent nucleoli are easily appreciated (Papanicolaou stain, $\times 400$ ). [Color figure can be viewed in the online issue, which is available at www.interscience. wiley.com.]

radiologic and clinical data, the diagnostic sensitivity, specificity, positive predictive values (PPV), negative predictive values (NPV) and accuracy were calculated. The diagnostic parameters for combined FNA with $\mathrm{BC}$ were compared with that for FNA or BC alone.

\section{Results}

A total of 97 patients who underwent simultaneous or consecutive FNA and BC were reviewed in this study. There were 55 men and 42 women with an average age of 62 (36-82) years old. Radiologic imaging detected a discrete mass in 72 patients among whom 64 had a mass in the head of the pancreas and the remaining eight had a pancreatic body/tail mass. The masses measured from 0.6 to $7.4 \mathrm{~cm}$, among which 64 were solid and eight were cystic. Imaging studies revealed pancreatiobillary duct dilation without definitive evidence of mass lesions in 25 patients. Both procedures were performed once in 69 patients and twice in five patients. Twenty-three patients underwent more than one BC or FNA. NO serious complications such as pancreatitis, bacteremia, perforation, hemorrhage, or bile peritonitis were documented in our data system. The diagnosis of NEG was upgraded to AN on repeating FNA (one patient) or BC (six patients). As shown in Table I, cytological evaluation of BC and/or FNA identified 42 cases as POS including 38 ductal adenocarcinomas, two endocrine tumors, one mucin-producing tumor, and one metastatic renal cell carcinoma; 15 cases as AN including 14 ductal adenocarcinoma and one mucin-producing tumor; and 40 cases as NEG with benign/reactive changes. $\mathrm{F} / \mathrm{U}$ with histopathology was 
JING ET AL.

Table I. Distribution of Diagnostic Categories and F/U Results

\begin{tabular}{llclc}
\hline Category & \multicolumn{1}{c}{$\begin{array}{c}\text { Cytologic diagnosis } \\
\text { (total number) }\end{array}$} & $\begin{array}{c}F N A / B C \\
\text { diagnosis }\end{array}$ & $\begin{array}{c}F / U \\
\text { diagnosis }\end{array}$ & $\begin{array}{c}F / U \text { by surgery/ } \\
\text { clinical outcome }\end{array}$ \\
\hline POS & Adenocarcinoma (38) & $33 / 14$ & Adenocarcinoma & $19 / 19$ \\
& Endocrine tumor (2) & $2 / 0$ & Endocrine tumor & $2 / 0$ \\
& Mucin-producing tumor (1) & $1 / 1$ & IPMN & $1 / 0$ \\
AN & Metastatic renal cell carcinoma (1) & $1 / 0$ & Metastatic renal cell carcinoma & $0 / 1$ \\
& Adecarcinoma (14) & $9 / 9$ & Adenocarcinoma & $10 / 2$ \\
& & & Chronic pancreatitis & $2 / 0$ \\
NEG & Mucin-producing tumor (1) & $0 / 1$ & IPMN & $1 / 0$ \\
& Benign or reactive (40) & $40 / 40$ & Adenocarcinoma & $8 / 1$ \\
& & & Serous cystadenoma & $1 / 0$ \\
& & & Chronic pancreatitis & $0 / 30$ \\
\hline
\end{tabular}

Table II. Correlation Between FNA and BC

\begin{tabular}{lrrrrr}
\hline & & \multicolumn{4}{c}{ BC diagnosis } \\
\cline { 3 - 6 } & & POS & AN & NEG & Total \\
\hline FNA diagnosis & POS & 10 & 8 & 19 & 37 \\
& AN & 1 & 4 & 5 & 10 \\
& NEG & 4 & 6 & 40 & 50 \\
& Total & 15 & 18 & 64 & 97 \\
\hline
\end{tabular}

available in 44 patients. Combined information on clinical course/outcome and radiological imaging were collected in $\mathrm{F} / \mathrm{U}$ of the remaining 53 patients. $\mathrm{F} / \mathrm{U}$ confirmed the cytological diagnoses for the POS cases. Thirteen of 15 patients with cytologic diagnoses of AN proved having ductal adenocarcinoma (12 patients) and intraductal papillary mucinous neoplasms (IPMN, one patient) and the remaining two patients having chronic pancreatitis (falsepositive) on surgical F/U. F/U also revealed that 10 of 40 patients diagnosed as NEG by both BC and FNA had a neoplasm (false-negative), including nine ductal adenocarcinomas and one serous cystadenoma. Cytologic re-review revealed that the two false-positive diagnoses and 10 false-negative diagnoses were made due to over interpretation of reactive atypia in chronic pancreatitis and sampling error, respectively.

Table II illustrates correlation between FNA and BC. Based on diagnoses of FNA and BC, the cohort of 97 patients was divided as follows: Twenty-three patients had concordant FNA and BC diagnoses of POS/AN. Thirty-four patients had disconcordant (POS/AN vs. NEG) FNA and BC diagnoses. Forty patients had negative diagnoses on both FNA and BC.

Overall, F/U confirmed the neoplasm in 65 patients and non-neoplastic conditions in 32 patients. FNA identified more positive cases (37) than BC (15). However, BC detected four POS and six AN cases whereas FNA gave false-negative results. Diagnostic sensitivity, specificity, PPV, NPV and accuracy for combination of FNA and $\mathrm{BC}$, as well as for FNA or $\mathrm{BC}$ alone are shown in Table III.
Table III. Diagnostic Efficiency of Combining FNA with BC versus FNA or BC Alone

\begin{tabular}{lccc}
\hline \%) & & & $F N A$ and \\
Sensitivity & 69.2 & $5 C$ & $B C$ \\
Specificity & 93.8 & 100 & 84.6 \\
PPV & 95.7 & 100 & 100 \\
NPV & 60.0 & 50.0 & 100 \\
Accuracy & 77.3 & 67.0 & 76.2 \\
\hline
\end{tabular}

\section{Discussion}

The United States is among the countries with considerable mortality due to malignant neoplasms of the pancreas which accounts for nearly 30,000 cancer-related deaths each year. In spite of the implementation of different modalities, ${ }^{12}$ prompt diagnosis of pancreatic neoplasms remain a significant challenge for the radiologist, gastroenterologist and pathologist alike. In our institution, EUSguided FNA and/or brush cytology through ERCP are major approaches for establishing a preoperative pathologic diagnosis of pancreatic neoplasms. For each of the 97 patients included in the current study, both EUSguided FNA and BC were methods of choice for the cytological evaluation of suspected pancreatic neoplasms. To our knowledge, there was limited data regarding combination of both FNA and BC for diagnosing pancreatic neoplasms. Although a relatively small scale investigation, the current study is the first and largest comparative study in which all cases included underwent both FNA and BC and $\mathrm{F} / \mathrm{U}$ was performed.

The current study differs from previous studies in that cytologic samples suspicious and positive for neoplasm were grouped together into the POS category. This was performed based on the results from previous studies that demonstrated that a suspicious cytologic diagnosis is more likely to indicate the presence rather than absence of the neoplasm. ${ }^{4,5,8,13,14}$ Likewise, F/U of all the suspicious cases in the current study confirmed the presence of neoplasm which supports the rationality of the categorization. 
F/U of a total of 15 patients in the AN group (13 by histology, two by clinical data) confirmed a neoplasm in 13 cases. The remaining two cases were diagnosed by FNA and F/U revealed chronic pancreatitis. This finding is consistent with previous observation by others that the category of atypia had a higher positive predictive value. ${ }^{1,5,14}$ On the other hand, another study showed that clinical $\mathrm{F} / \mathrm{U}$ of atypical cytologic diagnoses revealed malignancy in less than half of the cases. ${ }^{4,8}$ The inconsistency may result from the variations in diagnostic thresholds and level of stringency in applying the diagnostic criteria during the cytologic evaluation of the lesions. We agreed with other investigators that correlation between cytologic features and clinical/radiologic findings also play an important role during the cytologic evaluation of such lesions.

With regard to the patients who had a positive diagnosis on both EUS-guided FNA and BC, the following situations might be encountered: (1) Both procedures were performed within 24-48 hours, preliminary interpretation was atypical/favor neoplasm or suspicious for neoplasm and a definitive diagnosis of positive for neoplasm was rendered later on final evaluation. (2) $\mathrm{BC}$ is routinely performed at the time of ERCP. If ERCP was the first test performed and a EUS-guided FNA performed 24-hour later, the result of $\mathrm{BC}$ would not be available prior to the EUS being performed.

EUS-guided FNA and/or BC were repeated under the circumstances that a neoplasm is highly suspected clinically and/or radiologically and initial samples failed to confirm the clinical/radiological findings. Additional BC upgraded the diagnosis from NEG to AN in about $1 / 3$ of the patients who underwent multiple procedures. A similar finding has been observed by others. ${ }^{1}$ However, both FNA and BC failed to detect a neoplasm in 10 patients in whom further $\mathrm{F} / \mathrm{U}$ confirmed nine adenocarcinomas and one serous cystadenoma. Similar to the previous studies, ${ }^{2}$ sampling error was a key contributor to the false-negative results in the current study as second review of those cases confirmed original negative diagnoses.

Among the 65 patients with a pancreatic tumor confirmed by histology or radiologic imaging and/or clinical outcome, FNA and BC rendered a fairly straightforward diagnosis of neoplasm in 37 and 15 patients, respectively. FNA and BC generated a diagnosis of favoring neoplasm in about an equal number of patients. The vast majority of the tumors (59/65) detected by FNA or BC are pancreatic ductal adenocarcinoma. It appears that FNA is superior to $\mathrm{BC}$ in identifying ductal adenocarcinoma, endocrine and metastatic tumors although the latter two conditions were composed of few cases. It is worth noting that $\mathrm{BC}$ detected nine ductal adenocarcinomas and one mucin-producing tumor (four POS and six AN cases) whereas FNA gave false-negative results due to sampling error.
The current study demonstrates that BC and EUSguided FNA are almost equally specific for the diagnosis of neoplasms, particularly ductal adenocarcinoma although the latter had better diagnostic sensitivity and accuracy. The combination of EUS-guided FNA and BC did provide a superior diagnostic sensitivity and accuracy than the use of either one of the methods alone whereas specificity is not adversely affected. In spite of the improvement in NPV, it remains relatively low. Thus, the possibilities of ductal adenocarcinoma cannot be excluded based on a negative cytologic diagnosis and the diagnosis must be interpreted in conjunction with radiologic imaging and clinical data in such an instance.

Although the current study involved less numbers of patients compared to the previous studies, ${ }^{1-4} \mathrm{BC}$ alone achieved a compatible sensitivity, specificity, PPV, NPV, and accuracy in diagnosing ductal adenocarcinoma. The diagnostic sensitivity and accuracy of EUS-guided FNA were slightly better than that reported by Wittmann et al. ${ }^{15}$ and lower than that published by others. ${ }^{5,7-10,14}$ The difference may relate to the patient populations enrolled in the individual studies, size and location of the lesions and technical matters involved consequently in the procedures. Of the 65 patients with neoplasm, more than $50 \%$ of them had a mass less than $3 \mathrm{~cm}$. In this regard, a significant change in diagnostic accuracy (84-69\%) has been documented in FNA diagnosing pancreatic lesions $\geq 3 \mathrm{~cm}$ versus those $<3 \mathrm{~cm}^{16}$

In summary, both EUS-guided FNA and BC are valuable modalities in the preoperative diagnosis of pancreatic neoplasms, particularly ductal adenocarcinoma. When used in combination, the two modalities complement each other and achieve better diagnostic yield in pancreatic lesions than either FNA or BC alone. A negative cytologic diagnosis does not exclude the possibility of ductal adenocarcinoma and the diagnosis must be interpreted in conjunction with radiological and clinical findings.

\section{Acknowledgments}

The authors thank Claire Michael, M.D., for reviewing the manuscript and Brian Smola, C.T., for proof reading the manuscript.

\section{References}

1. Sheehan MM, Fraser A, Ravindran R, McAteer D. Bile duct brushings cytology-improving sensitivity of diagnosis using the ThinPrep technique: A review of 113 cases. Cytopathology 2007;18: 225-233.

2. Logrono R, Kurtycz DF, Molina CP, Trivedi VA, Wong JY, Block KP. Analysis of false-negative diagnoses on endoscopic brush cytology of biliary and pancreatic duct strictures: The experience at 2 university hospitals. Arch Pathol Lab Med 2000;124:387-392.

3. Kocjan G, Smith AN. Bile duct brushings cytology: Potential pitfalls in diagnosis. Diagn Cytopathol 1997;16:358-363. 


\section{JING ET AL.}

4. Volmar KE, Vollmer RT, Routbort MJ, Creager AJ. Pancreatic and bile duct brushing cytology in 1000 cases: Review of findings and comparison of preparation methods. Cancer 2006;108:231-238.

5. Shin HJ, Lahoti S, Sneige N. Endoscopic ultrasound-guided fineneedle aspiration in 179 cases: The M.D. Anderson Cancer Center experience. Cancer 2002;96:174-180.

6. Raut CP, Grau AM, Staerkel GA, et al. Diagnostic accuracy of endoscopic ultrasound-guided fine-needle aspiration in patients with presumed pancreatic cancer. J Gastrointest Surg 2003;7:118-126; discussion 127-128.

7. de Luna R, Eloubeidi MA, Sheffield MV, et al. Comparison of ThinPrep and conventional preparations in pancreatic fine-needle aspiration biopsy. Diagn Cytopathol 2004;30:71-76.

8. Mitsuhashi T, Ghafari S, Chang CY, Gu M. Endoscopic ultrasoundguided fine needle aspiration of the pancreas: Cytomorphological evaluation with emphasis on adequacy assessment, diagnostic criteria and contamination from the gastrointestinal tract. Cytopathology 2006; 17:34-41.

9. Yun SS, Remotti H, Vazquez MF, Crapanzano JP, Saqi A. Endoscopic ultrasound-guided biopsies of pancreatic masses: Comparison between fine needle aspirations and needle core biopsies. Diagn Cytopathol 2007;35:276-282.

10. Agarwal B, Krishna NB, Labundy JL, Safdar R, Akduman EI. EUS and/or EUS-guided FNA in patients with CT and/or magnetic resonance imaging findings of enlarged pancreatic head or dilated pan- creatic duct with or without a dilated common bile duct. Gastrointest Endosc 2008;68:237-242; quiz 334, 335.

11. Savides TJ, Donohue M, Hunt G, et al. EUS-guided FNA diagnostic yield of malignancy in solid pancreatic masses: A benchmark for quality performance measurement. Gastrointest Endosc 2007;66: 277-282.

12. DiMagno EP, Reber HA, Tempero MA. AGA technical review on the epidemiology, diagnosis, and treatment of pancreatic ductal adenocarcinoma. American Gastroenterological Association. Gastroenterology 1999;117:1464-1484.

13. Basir Z, Pello N, Dayer AM, Shidham VB, Komorowski RA. Accuracy of cytologic interpretation of pancreatic neoplasms by fine needle aspiration and pancreatic duct brushings. Acta Cytol 2003;47:733-738.

14. Eloubeidi MA, Jhala D, Chhieng DC, et al. Yield of endoscopic ultrasound-guided fine-needle aspiration biopsy in patients with suspected pancreatic carcinoma. Cancer 2003;99:285-292.

15. Wittmann J, Kocjan G, Sgouros SN, Deheragoda M, Pereira SP. Endoscopic ultrasound-guided tissue sampling by combined fine needle aspiration and trucut needle biopsy: A prospective study. Cytopathology 2006; 17:27-33.

16. Mallery JS, Centeno BA, Hahn PF, Chang Y, Warshaw AL, Brugge WR. Pancreatic tissue sampling guided by EUS, CT/US, and surgery: A comparison of sensitivity and specificity Gastrointest Endosc 2002;56:218-224. 